\title{
COMPACTNESS CRITERIA FOR THE RESOLVENT OF THE FOKKER-PLANCK OPERATOR
}

\author{
WEI-XI LI
}

\begin{abstract}
In this paper we study the spectral property of a Fokker-Planck operator with potential. By virtue of a multiplier method inspired by Nicolas Lerner, we obtain new compactness criteria for its resolvent, involving the control of the positive eigenvalues of the Hessian matrix of the potential.
\end{abstract}

\section{INTRODUCTION AND MAIN RESULTS}

The Fokker-Planck operator reads

$$
P=y \cdot \partial_{x}-\partial_{x} V(x) \cdot \partial_{y}-\Delta_{y}+\frac{|y|^{2}}{4}-\frac{n}{2}, \quad(x, y) \in \mathbb{R}^{2 n},
$$

where $x$ denotes the space variable and $y$ denotes the velocity variable, and $V(x)$ is a potential defined in the whole spatial space $\mathbb{R}_{x}^{n}$. In this work we are mainly concerned with the compact resolvent property for the non-selfadjoint Fokker-Planck operator, and this is motivated by a conjecture stated by Helffer and Nier (see [7, Conjecture 1.2]), which reveals the close link between the compact resolvent property for the Fokker-Planck operator and the same property for the corresponding Witten Laplacian. Precisely,

Conjecture 1.1 (Helffer-Nier's Conjecture). The Fokker-Planck operator $P$ has a compact resolvent if and only if the Witten Laplacian $\Delta_{V / 2}^{(0)}$, defined by

$$
\Delta_{V / 2}^{(0)}=-\Delta_{x}+\frac{1}{4}\left|\partial_{x} V(x)\right|^{2}-\frac{1}{2} \Delta_{x} V(x),
$$

has a compact resolvent.

The necessity part, that the Witten Laplacian $\Delta_{V / 2}^{(0)}$ has a compact resolvent if the Fokker-Planck operator $P$ is with compact resolvent, has already established by Helffer and Nier (c.f. 17, Theorem 1.1]). The reverse implication still remains open up to now for general potential, and it is indeed valid under some conditions on the potential $V$. For instance, following the analysis in [7, 13] with some improvements, the author ([17]) proved that if $V$ satisfies that

$$
\forall|\alpha|=2, \exists C_{\alpha}>0, \quad\left|\partial_{x}^{\alpha} V(x)\right| \leq C_{\alpha}\left\langle\partial_{x} V(x)\right\rangle^{s} \quad \text { with } \mathrm{s}<\frac{4}{3},
$$

then Fokker-Planck operator has a compact resolvent provided the Witten-Laplacian has a compact resolvent or $\lim _{|x| \rightarrow+\infty}\left|\partial_{x} V(x)\right|=+\infty$, and moreover a constant $C$ exists such that the following weighted estimate

$$
\left\|\left|\partial_{x} V(x)\right|^{2 / 3} u\right\|_{L^{2}} \leq C\left(\|P u\|_{L^{2}}+\|u\|_{L^{2}}\right)
$$

holds for all $u \in C_{0}^{\infty}\left(\mathbb{R}^{2 n}\right)$. Here and throughout the paper we will use the notation

$$
\langle\cdot\rangle=\left(1+|\cdot|^{2}\right)^{1 / 2}
$$

2010 Mathematics Subject Classification. Primary 35H10; Secondary 47A10.

Key words and phrases. Compact resolvent, Fokker-Planck operator, Witten Laplacian. 
which is equivalent to the Modulus $|\cdot|$, and use $\|\cdot\|_{L^{2}}$ and $\langle\cdot, \cdot\rangle_{L^{2}}$ to denote respectively the norm and inner product of the complex Hilbert space $L^{2}\left(\mathbb{R}^{2 n}\right)$, and denote by $C_{0}^{\infty}\left(\mathbb{R}^{2 n}\right)$ the set of smooth compactly supported functions.

We remark the drawback of the condition (2) is that it doesn't give any information for the dependence on the sign of $V$, which plays import role in the analysis of compact resolvent property for Witten Laplacian. For instance it is well-known (see [7, 18]) that the Witten Laplacian $\Delta_{V / 2}^{(0)}$ with $V=-x_{1}^{2} x_{2}^{2}$ has a compact resolvent, while 0 actually belongs to the essential spectrum of Witten Laplacian $\Delta_{V / 2}^{(0)}$ with $V=x_{1}^{2} x_{2}^{2}$ and thus its resolvent cannot be compact. By the general criteria for Schrödinger operators we see if

$$
\frac{1}{4}\left|\partial_{x} V(x)\right|^{2}-\frac{1}{2} \Delta_{x} V \rightarrow+\infty, \text { as }|x| \rightarrow+\infty,
$$

or more generally (see [7, Proposition 3.1] for instance), if

$$
\frac{t}{4}\left|\partial_{x} V(x)\right|^{2}-\frac{1}{2} \Delta_{x} V \rightarrow+\infty, \text { as }|x| \rightarrow+\infty
$$

for some $t \in] 0,2\left[\right.$, then the Witten Laplacian $\Delta_{V / 2}^{(0)}$ has a compact resolvent. We refer the reader to [7] for other criteria presented with detailed discussion. These criteria show the microlocal property, i.e., the dependence on the sign of $V$, for the compact resolvent of Witten Laplacian. As far as Fokker-Planck operator is concerned, Helffer-Nier's Conjecture suggests strongly it should have the similar microlocal property as the Witten Laplacian. And this kind of dependence property for Fokker-Planck operator is not clear by now. In the present work we will give some sufficient conditions for the compact resolvent of Fokker-Planck operator, mainly based on the sign of the eigenvalues of the Hessian matrix $\left(\partial_{x_{i} x_{j}} V\right)_{1 \leq i, j \leq n}$. Our results can be stated as follows.

Theorem 1.2. Denote by $\lambda_{\ell}(x), 1 \leq \ell \leq n$, the eigenvalues of the Hessian matrix

$$
\left(\partial_{x_{i} x_{j}} V(x)\right)_{1 \leq i, j \leq n} \text {. }
$$

With each $x \in \mathbb{R}^{n}$ we associate a set $I_{x}$ of indexes defined by

$$
I_{x}=\left\{1 \leq \ell \leq n ; \lambda_{\ell}(x)>0\right\} .
$$

Suppose that there exists a constant $C$ such that

$$
\forall x \in \mathbb{R}^{n}, \quad \sum_{j \in I_{x}} \lambda_{j}(x) \leq C\left\langle\partial_{x} V(x)\right\rangle^{4 / 3} .
$$

Then the following conclusions hold.

(i) There exists a constant $C_{*}$ such that

$$
\forall u \in C_{0}^{\infty}\left(\mathbb{R}^{2 n}\right), \quad\left\|\left|\partial_{x} V(x)\right|^{1 / 16} u\right\|_{L^{2}} \leq C_{*}\left(\|P u\|_{L^{2}}+\|u\|_{L^{2}}\right) .
$$

As a result, the Fokker-Planck operator $P$ has a compact resolvent if

$$
\lim _{|x| \rightarrow+\infty}\left|\partial_{x} V(x)\right|=+\infty .
$$

(ii) Suppose there exists a number $\alpha \geq 0$, such that

$$
\lim _{|x| \rightarrow+\infty}\left(\alpha\left|\partial_{x} V(x)\right|^{2}-\Delta_{x} V(x)\right)=+\infty .
$$

Then we can find a constant $\tilde{C}$, depending on $\alpha$, such that

$$
\forall u \in C_{0}^{\infty}\left(\mathbb{R}^{2 n}\right), \quad\left\|\left.|\alpha| \partial_{x} V(x)\right|^{2}-\left.\Delta_{x} V(x)\right|^{1 / 80} u\right\|_{L^{2}} \leq \tilde{C}\left(\|P u\|_{L^{2}}+\|u\|_{L^{2}}\right),
$$

and thus the Fokker-Planck operator $P$ has a compact resolvent as a result. 
The assumption (44) is an improvement of the condition (21). We mention that the index $4 / 3$ in (44) is not sharp, and the following Theorem 1.3 and Corollary 1.4 are devoted to showing a better index 14/5 may be expected.

Theorem 1.3. Suppose that there exists a number $\tau \geq 0$, such that the matrix

$$
A_{\tau}(x)=\left(a_{i j}^{\tau}(x)\right)_{1 \leq i, j \leq n}, \quad a_{i j}^{\tau}=\tau\left\langle\partial_{x} V\right\rangle^{\frac{4}{5}}\left(\partial_{x_{i}} V\right)\left(\partial_{x_{j}} V\right)-\partial_{x_{i} x_{j}} V+\tau \delta_{i j}
$$

is positive-definite for all $x \in \mathbb{R}^{n}$, where $\delta_{i j}$ is the Kronecker Delta. Then there is a constant $C$, such that

$$
\forall u \in C_{0}^{\infty}\left(\mathbb{R}^{2}\right), \quad\left\|\left|\partial_{x} V(x)\right|^{1 / 20} u\right\|_{L^{2}}+\sum_{1 \leq i, j \leq n}\left\|\left|a_{i j}^{\tau}(x)\right|^{1 / 80} u\right\|_{L^{2}} \leq C\left(\|P u\|_{L^{2}}+\|u\|_{L^{2}}\right) .
$$

As a result, the Fokker-Planck operator $P$ has a compact resolvent if

$$
\lim _{|x| \rightarrow+\infty}\left(\left|\partial_{x} V(x)\right|+\sum_{1 \leq i, j \leq n}\left|a_{i j}^{\tau}(x)\right|\right)=+\infty .
$$

As an immediate consequence, when $n=1$ we have the compactness criteria for Fokker-Planck operator, which is an improvement of the corresponding condition (3) for Witten Laplacian. Precisely,

Corollary 1.4. Let $n=1$ and let $V(x) \in C^{2}(\mathbb{R})$. Suppose that there exists $\tau \geq 0$, such that

$$
\lim _{|x| \rightarrow+\infty} \tau\left|\partial_{x} V(x)\right|^{14 / 5}-\Delta_{x} V(x)=+\infty .
$$

Then the Fokker-Planck operator $P$ has a compact resolvent.

Remark 1.5. In the special case when $n=1$, using Corollary 1.4 and the necessity part in HelfferNier Conjecture (c.f. [7, Theorem 1.1]), we can also improve the criteria (3) for Witten Laplacian, by allowing $t$ to range over $[0,+\infty[$ instead of $] 0,2[$ and relaxing the index 2 there by $14 / 5$.

Remark 1.6. The hypotheses in Theorem 1.2 and Theorem [1.3, are related to the sign of the eigenvalues of the Hessian matrix $\left(\partial_{x_{i} x_{j}} V\right)_{1 \leq i, j \leq n}$. In fact these assumptions are obviously fulfilled when the Hessian matrix is negative-semidefinite. When the Hessian matrix is positive-semidefinite or indefinite, we requires that the positive eigenvalues of Hessian matrix, instead of all the second derivatives in the condition (2), are dominated by $\left\langle\partial_{x} V\right\rangle^{4 / 3}$. Now look back at the aforementioned potential $V= \pm x_{1}^{2} x_{2}^{2}$, and it is clear that these hypotheses are fulfilled by $V=-x_{1}^{2} x_{2}^{2}$ and violated by $V=x_{1}^{2} x_{2}^{2}$.

Remark 1.7. In [7, 8], the authors introduced a compactness criterion for Witten Laplacian with polynomial potential $V$, based on the group theory. And it is also natural and interesting to expect the similar group theoretical compactness criteria for Fokker-Planck operator. Now consider such a potential, not necessary to be a polynomial, that the matrix

$$
\tilde{A}_{\tau}(x)=\left(\tilde{a}_{i j}^{\tau}(x)\right)_{1 \leq i, j \leq n}, \quad \tilde{a}_{i j}^{\tau}(x)=\tau\left|\partial_{x} V(x)\right|^{4 / 5}\left(\partial_{x_{i}} V(x)\right)\left(\partial_{x_{j}} V(x)\right)-\partial_{x_{i} x_{j}} V(x)
$$

is positive-semidefinite for some $\tau \geq 0$. This condition is slightly stronger than the one in Theorem 1.3. and it yields

$$
-\Delta_{x} V(x)+\tau\left|\partial_{x} V(x)\right|^{14 / 5} \geq 0 .
$$

Thus repeating the arguments used to prove maximum principle for elliptic equations, we see $V$ doesn't have local minimum in $\mathbb{R}_{x}^{n}$, except the constant-valued potentials. So this kind of microlocal property is imposed directly on the potential rather than its "limiting polynomials" in the sense of [7, 8. 
Due to the lack of estimates on the higher derivatives of $V$, we can't follow the global symbolic calculus to prove our results, although this method is efficiently explored to investigate the hypoellipticity and the compact resolvent of Fokker-Planck operator (c.f. [7, 13]). Instead we will use a multiplier method inspired by N. Lerner (see for instance [14, 15] and references therein), which is based on the Poisson bracket analysis for the real and imaginary parts of the Fokker-Planck operator. We hope this method not only applies to analyze the weighted estimate and the compact resolvent, but also may give insights on the sign conditions to investigate the subellipticity (see [4, 5, 1, 13, 17, 19] for instance) of Fokker-Planck operator.

We end up the introduction by mentioning that as a diffusive models, the study of Fokker-Planck equation is of independent interest in kinetic theory and nonequilibrium statistical physics. Here one of the basic problems is to analyze the large time behavior of solutions to the time-dependent FokkerPlanck equation and prove that these solutions converge exponentially towards the equilibrium as $t$ goes to $+\infty$. Various approaches, such as hypoellipticity, hypocoercivity, entropy method and so on, are developed to study this problem, and satisfactory results are achieved. We refer to [6, 7, 9, 10, 11, 13, 17, 20] and references therein for more detail and [2] for the spectral analysis on the non-selfadjoint Schrödinger operators with compact resolvent. Finally we remark that in order to study the exponentially trend problem, an efficient method is to investigate the spectral gap, which is usually reduced to analyze the compactness of resolvent. On the other hand, when the Fokker-Planck operator has an essential spectrum, only polynomial convergence rate is expected, see the recent work [21] for the study on short-range potentials.

Acknowledgements. We are grateful to the referee for the valuable suggestions which helped the author correct several mistakes. Part of the work was done when the author visited The Institute of Mathematical Sciences, The Chinese University of Hong Kong, and he would like to thank their support and hospitality. We also appreciate the support from NSF of China(11422106) and Fok Ying Tung Education Foundation (151001).

\section{Proof the main Results}

We firstly list some notations and facts used throughout the paper. The proofs of the main results, Theorem 1.2 and Theorem [1.3, are presented in Subsection 2.1 and Subsection 2.2, where two multipliers $\mathcal{M}$ and $\mathcal{K}$ (see Lemma 2.1 and Lemma 2.2 below) are introduced respectively. This kind of multiplier method is inspired by N. Lerner [16], and here it means that we have to choose carefully an operator $M$ (multiplier) which is bounded and self-adjoint in $L^{2}$ space, such that

$$
\operatorname{Re}\left\langle\left(y \cdot \partial_{x}-\partial_{x} V(x) \cdot \partial_{y}\right) u, M u\right\rangle_{L^{2}}
$$

has a good lower bound (weighted estimate here) on one side, and on the other side,

$$
\left|\left\langle\left(-\Delta_{y}+|y|^{2} / 4-n / 2\right) u, M u\right\rangle_{L^{2}}\right|
$$

is bounded from above by $\|P u\|_{L^{2}}+\|u\|_{L^{2}}$. The multipliers chosen here are motivated by the Poisson bracket analysis for the real and imaginary parts of symbol for the Fokker-Planck operator. Precisely, if we denoted by $\left[Q_{1}, Q_{2}\right]$ the commutator between two operators $Q_{1}$ and $Q_{2}$, which is defined by

$$
\left[Q_{1}, Q_{2}\right]=Q_{1} Q_{2}-Q_{2} Q_{1},
$$

and also use the notation that $X_{0}=y \cdot \partial_{x}-\partial_{x} V(x) \cdot \partial_{y}$ and $X_{j}=\partial_{y_{j}}+\frac{y_{j}}{2}, j=1, \cdots n$, then we can rewrite the Fokker-Planck operator $P$ define in (1) as

$$
P=X_{0}+\sum_{j=1}^{n} X_{j}^{*} X_{j},
$$


and moreover we compute

$$
\left[X_{0}, \sum_{j=1}^{n} X_{j}^{*} X_{j}\right]=-\frac{1}{2} \partial_{x} V \cdot y+2 \partial_{x} \cdot \partial_{y}
$$

and

$$
\left[X_{0},\left[X_{0}, \sum_{j=1}^{n} X_{j}^{*} X_{j}\right]\right]=-2 \Delta_{x}+\frac{1}{2}\left|\partial_{x} V\right|^{2}-\frac{1}{2} \sum_{1 \leq i, j \leq n}\left(\partial_{x_{i} x_{j}} V\right) y_{i} y_{j}+2 \sum_{1 \leq i, j \leq n}\left(\partial_{x_{i} x_{j}} V\right) \partial_{y_{i}} \partial_{y_{j}} .
$$

Thus the properties of subelliptic and weighted estimates in $x$ variable can be deduced from the commutator above if some kind of conditions (negative semi-definite for instance) are imposed on the Hessian matrix $\left(\partial_{x_{i} x_{j}} V\right)_{1 \leq i, j \leq n}$. This suggests that the multipliers $\mathcal{M}$ and $\mathcal{K}$ here (see Lemma 2.1 and Lemma 2.2 below) should be chosen through the first-commutator analysis in (7). In this work we will investigate only the weighted estimate and thus the essential part in the multipliers is the term $\partial_{x} V \cdot y$ in (17). Moreover it seems reasonable that the term $\partial_{x} \cdot \partial_{y}$ in (7) shouldn't be involved in the multipliers, since it corresponds to the hypoellipticity and thus more estimates on the higher derivatives of $V$ are required rather than the ones of second order. We refer to [1, 12] for the multipliers introduced to deduce the hypoellipticity of kinetic operators.

Next we will give some estimates to be used frequently. Observe $X_{0}$ is an anti-selfadjoint operator in $L^{2}$ and thus it is clear that

$$
\forall u \in C_{0}^{\infty}\left(\mathbb{R}^{2 n}\right), \quad \sum_{j=1}^{n}\left\|X_{j} u\right\|_{L^{2}} \leq\|\langle y\rangle u\|_{L^{2}}+\sum_{1 \leq j \leq n}\left\|\partial_{y_{j}} u\right\|_{L^{2}} \leq C\left(\|P u\|_{L^{2}}+\|u\|_{L^{2}}\right) .
$$

We will use the following result which is just a consequence of Hörmander's bracket condition (cf. [3] for instance), i.e., a constant $C$ exists such that for any vector-valued function $\theta(x)=$ $\left(\theta_{1}(x), \cdots, \theta_{n}(x)\right)$ of $x$ variable and for any $v \in C_{0}^{\infty}\left(\mathbb{R}_{y}^{n}\right)$, we have

$$
\left\|\left|\theta_{k}(x)\right|^{1 / 2} v\right\|_{L^{2}\left(\mathbb{R}_{y}^{n}\right)} \leq C\left(\|(\theta(x) \cdot y) v\|_{L^{2}\left(\mathbb{R}_{y}^{n}\right)}+\left\|\partial_{y_{k}} v\right\|_{L^{2}\left(\mathbb{R}_{y}^{n}\right)}\right) .
$$

2.1. Proof of Theorem 1.2. We prove in this subsection Theorem 1.2. To do so we begin with the following estimate which holds for quite general potential.

Lemma 2.1. Let $V(x) \in C^{2}\left(\mathbb{R}^{n}\right)$. Then for all $\left.\sigma \in\right] 0,1\left[\right.$, there exists a constant $C_{\sigma}>0$ such that for any $u \in C_{0}^{\infty}\left(\mathbb{R}^{2 n}\right)$ we have

$$
\begin{gathered}
\sigma\left\langle\frac{\left(1+|y|^{8}\right)\left\langle\partial_{x} V(x)\right\rangle^{2}}{\rho^{3}} \frac{y^{2}}{1+y^{2}} u, u\right\rangle_{L^{2}}+\left\langle\frac{\rho}{\langle y\rangle^{4}} u, u\right\rangle_{L^{2}} \\
-\sum_{1 \leq i, j \leq n}\left\langle\frac{\left(1+|y|^{8}\right)\left(\partial_{x_{i} x_{j}} V\right) y_{i} y_{j}}{\rho^{3}} \frac{y^{2}}{1+y^{2}} u, u\right\rangle_{L^{2}} \\
\leq C_{\sigma}\left(\|P u\|_{L^{2}}^{2}+\|u\|_{L^{2}}^{2}\right),
\end{gathered}
$$

where the function $\rho \in C^{1}\left(\mathbb{R}^{2 n}\right)$ is defined by

$$
\rho=\rho(x, y)=\left(1+|y|^{8}+\left|\partial_{x} V(x) \cdot y\right|^{2}\right)^{\frac{1}{2}} .
$$

Proof. To simplify the notations we will use $C$ in the proof to denote different constants, and similarly use $C_{\varepsilon}$ to denote different constants depending on $\varepsilon$. This lemma is to be proven by the multiplier method. Firstly we introduce a multiplier $\mathcal{M}$, which is a $C^{1}\left(\mathbb{R}^{2 n}\right)$ function defined by

$$
\mathcal{M}=\mathcal{M}(x, y)=\frac{2 \partial_{x} V(x) \cdot y}{\rho(x, y)} \frac{y^{2}}{1+y^{2}}
$$


with $\rho$ given in Lemma 2.1. Recall $X_{0}=y \cdot \partial_{x}-\partial_{x} V(x) \cdot \partial_{y}$. Then using the relation

$$
\frac{1}{2}\left[\mathcal{M}, X_{0}\right]=\frac{\partial_{x} V(x) \cdot y}{\rho(x, y)}\left[\frac{y^{2}}{1+y^{2}}, X_{0}\right]+\left[\frac{\partial_{x} V(x) \cdot y}{\rho(x, y)}, X_{0}\right] \frac{y^{2}}{1+y^{2}},
$$

we calculate

$$
\begin{aligned}
\frac{1}{2}\left[\mathcal{M}, X_{0}\right]= & \frac{2\left|\partial_{x} V(x) \cdot y\right|^{2}}{\rho(x, y)\left(1+y^{2}\right)^{2}}+\frac{\left(1+|y|^{8}\right)\left|\partial_{x} V(x)\right|^{2}}{\rho^{3}} \frac{y^{2}}{1+y^{2}} \\
& -\frac{\left(1+|y|^{8}\right) \sum_{1 \leq i, j \leq n}\left(\partial_{x_{i} x_{j}} V\right) y_{i} y_{j}}{\rho^{3}} \frac{y^{2}}{1+y^{2}} \\
& -\frac{4\left|\partial_{x} V(x) \cdot y\right|^{2}|y|^{6}}{\rho^{3}} \frac{y^{2}}{1+y^{2}} .
\end{aligned}
$$

As a result, observe

$$
\operatorname{Re}\left\langle X_{0} u, \mathcal{M} u\right\rangle_{L^{2}}=\frac{1}{2}\left\langle\left[\mathcal{M}, X_{0}\right] u, u\right\rangle_{L^{2}},
$$

and thus

$$
\begin{aligned}
\operatorname{Re}\left\langle X_{0} u, \mathcal{M} u\right\rangle_{L^{2}}= & \left\langle\frac{\left(1+|y|^{8}\right)\left|\partial_{x} V(x)\right|^{2}}{\rho^{3}} \frac{y^{2}}{1+y^{2}} u, u\right\rangle_{L^{2}}+\left\langle\frac{2\left|\partial_{x} V(x) \cdot y\right|^{2}}{\rho(x, y)\left(1+y^{2}\right)^{2}} u, u\right\rangle_{L^{2}} \\
& -\left\langle\frac{\left(1+|y|^{8}\right) \sum_{1 \leq i, j \leq n}\left(\partial_{x_{i} x_{j}} V\right) y_{i} y_{j}}{\rho^{3}} \frac{y^{2}}{1+y^{2}} u, u\right\rangle_{L^{2}} \\
& -\left\langle\frac{4\left|\partial_{x} V(x) \cdot y\right|^{2}|y|^{6}}{\rho^{3}} \frac{y^{2}}{1+y^{2}} u, u\right\rangle_{L^{2}} .
\end{aligned}
$$

This, along with the inequalities that

$$
\begin{aligned}
\left\langle\frac{2 \rho}{\left(1+y^{2}\right)^{2}} u, u\right\rangle_{L^{2}} & =\left\langle\frac{2\left|\partial_{x} V(x) \cdot y\right|^{2}}{\rho(x, y)\left(1+y^{2}\right)^{2}} u, u\right\rangle_{L^{2}}+\left\langle\frac{2\left(1+|y|^{8}\right)}{\rho(x, y)\left(1+y^{2}\right)^{2}} u, u\right\rangle_{L^{2}} \\
& \leq\left\langle\frac{2\left|\partial_{x} V(x) \cdot y\right|^{2}}{\rho(x, y)\left(1+y^{2}\right)^{2}} u, u\right\rangle_{L^{2}}+C\|u\|_{L^{2}}^{2}
\end{aligned}
$$

and

$$
\left|\left\langle\frac{4\left|\partial_{x} V(x) \cdot y\right|^{2}|y|^{6}}{\rho^{3}} \frac{y^{2}}{1+y^{2}} u, u\right\rangle_{L^{2}}\right| \leq C\|\langle y\rangle u\|_{L^{2}}^{2} \leq C\left(\|P u\|_{L^{2}}^{2}+\|u\|_{L^{2}}^{2}\right),
$$

yields

$$
\begin{aligned}
&\left\langle\frac{\left(1+|y|^{8}\right)\left|\partial_{x} V(x)\right|^{2}}{\rho^{3}} \frac{y^{2}}{1+y^{2}} u, u\right\rangle_{L^{2}}+\left\langle\frac{2 \rho}{\left(1+y^{2}\right)^{2}} u, u\right\rangle_{L^{2}} \\
&-\left\langle\frac{\left(1+|y|^{8}\right) \sum_{1 \leq i, j \leq n}\left(\partial_{x_{i} x_{j}} V\right) y_{i} y_{j}}{\rho^{3}} \frac{y^{2}}{1+y^{2}} u, u\right\rangle_{L^{2}} \\
& \leq \operatorname{Re}\left\langle X_{0} u, \mathcal{M} u\right\rangle_{L^{2}}+C\left(\|P u\|_{L^{2}}^{2}+\|u\|_{L^{2}}^{2}\right) .
\end{aligned}
$$

On the other hand, since $\mathcal{M} \in L^{\infty}\left(\mathbb{R}^{2 n}\right)$ with $\|\mathcal{M}\|_{L^{\infty}} \leq 2$ then it is easy to see

$$
\left|\operatorname{Re}\langle P u, \mathcal{M} u\rangle_{L^{2}}\right| \leq\|P u\|_{L^{2}}^{2}+\|u\|_{L^{2}}^{2} .
$$


Recall $X_{j}=\partial_{y_{j}}+\frac{y_{j}}{2}, j=1, \cdots n$. Then direct computation gives, for each $1 \leq j \leq n$,

$$
\begin{aligned}
{\left[\mathcal{M}, X_{j}\right]=} & -\frac{2\left(1+|y|^{8}\right)\left(\partial_{x_{j}} V(x)\right)}{\rho^{3}} \frac{y^{2}}{\left(1+y^{2}\right)}+\frac{8\left(\partial_{x} V(x) \cdot y\right)|y|^{6} y_{j}}{\rho^{3}} \frac{y^{2}}{\left(1+y^{2}\right)} \\
& -\frac{4\left(\partial_{x} V(x) \cdot y\right) y_{j}}{\rho(x, y)\left(1+y^{2}\right)^{2}} .
\end{aligned}
$$

Thus, for any $\varepsilon>0$,

$$
\begin{aligned}
& \left|\operatorname{Re}\left\langle X_{j} u,\left[\mathcal{M}, X_{j}\right] u\right\rangle_{L^{2}}\right| \\
\leq & \varepsilon\left\|\rho^{-3}\left(1+y^{2}\right)^{-1}\left(1+|y|^{8}\right) y^{2}\left|\partial_{x_{j}} V(x)\right| u\right\|_{L^{2}}^{2}+C_{\varepsilon}\left(\left\|X_{j} u\right\|_{L^{2}}^{2}+\|u\|_{L^{2}}^{2}\right) \\
\leq & \varepsilon\left\langle\frac{\left(1+|y|^{8}\right)\left|\partial_{x_{j}} V(x)\right|^{2}}{\rho^{3}} \frac{y^{2}}{1+y^{2}} u, u\right\rangle_{L^{2}}+C_{\varepsilon}\left(\left\|X_{j} u\right\|_{L^{2}}^{2}+\|u\|_{L^{2}}^{2}\right) .
\end{aligned}
$$

This gives, using $\|\mathcal{M}\|_{L^{\infty}} \leq 2$ and (8),

$$
\begin{aligned}
\left|\operatorname{Re}\left\langle X_{j}^{*} X_{j} u, \mathcal{M} u\right\rangle_{L^{2}}\right| & \leq\left|\operatorname{Re}\left\langle X_{j} u, \mathcal{M} X_{j} u\right\rangle_{L^{2}}\right|+\left|\operatorname{Re}\left\langle X_{j} u,\left[\mathcal{M}, X_{j}\right] u\right\rangle_{L^{2}}\right| \\
& \leq \varepsilon\left\langle\frac{\left(1+|y|^{8}\right)\left|\partial_{x_{j}} V(x)\right|^{2}}{\rho^{3}} \frac{y^{2}}{1+y^{2}} u, u\right\rangle_{L^{2}}+C_{\varepsilon}\left(\left\|X_{j} u\right\|_{L^{2}}^{2}+\|u\|_{L^{2}}^{2}\right) \\
& \leq \varepsilon\left\langle\frac{\left(1+|y|^{8}\right)\left|\partial_{x_{j}} V(x)\right|^{2}}{\rho^{3}} \frac{y^{2}}{1+y^{2}} u, u\right\rangle_{L^{2}}+C_{\varepsilon}\left(\|P u\|_{L^{2}}^{2}+\|u\|_{L^{2}}^{2}\right) .
\end{aligned}
$$

Then

$$
\sum_{j=1}^{n}\left|\operatorname{Re}\left\langle X_{j}^{*} X_{j} u, \mathcal{M} u\right\rangle_{L^{2}}\right| \leq \varepsilon\left\langle\frac{\left(1+|y|^{8}\right)\left|\partial_{x} V(x)\right|^{2}}{\rho^{3}} \frac{y^{2}}{1+y^{2}} u, u\right\rangle_{L^{2}}+C_{\varepsilon}\left(\|P u\|_{L^{2}}^{2}+\|u\|_{L^{2}}^{2}\right) .
$$

Consequently, from (10), (11) and the relationship

$$
\operatorname{Re}\left\langle X_{0} u, \mathcal{M} u\right\rangle_{L^{2}}=\operatorname{Re}\langle P u, \mathcal{M} u\rangle_{L^{2}}-\operatorname{Re} \sum_{j=1}^{n}\left\langle X_{j}^{*} X_{j} u, \mathcal{M} u\right\rangle_{L^{2}},
$$

it follows that

$$
\begin{gathered}
\left\langle\frac{\left(1+|y|^{8}\right)\left|\partial_{x} V(x)\right|^{2}}{\rho^{3}} \frac{y^{2}}{1+y^{2}} u, u\right\rangle_{L^{2}}+\left\langle\frac{2 \rho}{\left(1+y^{2}\right)^{2}} u, u\right\rangle_{L^{2}} \\
-\left\langle\frac{\left(1+|y|^{8}\right) \sum_{1 \leq i, j \leq n}\left(\partial_{x_{i} x_{j}} V\right) y_{i} y_{j}}{\rho^{3}} \frac{y^{2}}{1+y^{2}} u, u\right\rangle_{L^{2}} \\
\leq \varepsilon\left\langle\frac{\left(1+|y|^{8}\right)\left|\partial_{x} V(x)\right|^{2}}{\rho^{3}} \frac{y^{2}}{1+y^{2}} u, u\right\rangle_{L^{2}}+C_{\varepsilon}\left(\|P u\|_{L^{2}}^{2}+\|u\|_{L^{2}}^{2}\right) .
\end{gathered}
$$

As the result, for all $\sigma$ with $0<\sigma<1$, letting $\varepsilon=1-\sigma$ gives the desired estimate in Lemma 2.1] The proof is thus complete.

The rest part is devoted to the proof of Theorem 1.2 .

Proof of Theorem 1.2. For the symmetric Hessian matrix $\left(\partial_{x_{i} x_{j}} V\right)_{1 \leq i, j \leq n}$, we can find a $n \times n$ orthogonal matrix $Q(x)=\left(q_{i j}(x)\right)_{1 \leq i, j \leq n}$ such that

$$
Q^{T}\left(\begin{array}{cccc}
\lambda_{1} & & & \\
& \lambda_{2} & & \\
& & \ddots & \\
& & & \lambda_{n}
\end{array}\right) Q=\left(\partial_{x_{i} x_{j}} V\right)_{1 \leq i, j \leq n}
$$


where $\lambda_{j}, 1 \leq j \leq n$ are the eigenvalues of the Hessian $\left(\partial_{x_{i} x_{j}} V\right)_{1 \leq i, j \leq n}$. Then for any $x \in \mathbb{R}^{n}$ we can write

$$
-\sum_{j \notin I_{x}} \lambda_{j}(x)\left[(Q(x) y)_{j}\right]^{2}=-\sum_{1 \leq i, j \leq n}\left(\partial_{x_{i} x_{j}} V(x)\right) y_{i} y_{j}+\sum_{j \in I_{x}} \lambda_{j}(x)\left[(Q(x) y)_{j}\right]^{2},
$$

where $(Q(x) y)_{j}$ stands for the $j$-th component of the vector $Q(x) y$, and

$$
I_{x}=\left\{1 \leq \ell \leq n ; \lambda_{\ell}(x)>0\right\} .
$$

Thus it follows from (13) and the assumption (41) that, for any $x \in \mathbb{R}^{n}$,

$$
\sum_{j \notin I_{x}}\left(-\lambda_{j}(x)\right)\left[(Q(x) y)_{j}\right]^{2} \leq-\sum_{1 \leq i, j \leq n}\left(\partial_{x_{i} x_{j}} V(x)\right) y_{i} y_{j}+C\left\langle\partial_{x} V(x)\right\rangle^{4 / 3}|y|^{2} .
$$

This together with the estimate in Lemma 2.1 yields, for all $0<\sigma<1$ and for any $\varepsilon>0$,

$$
\begin{aligned}
& \sigma\left\langle\frac{\left(1+|y|^{8}\right)\left\langle\partial_{x} V(x)\right\rangle^{2}}{\rho^{3}} \frac{y^{2}}{1+y^{2}} u, u\right\rangle_{L^{2}}+\left\langle\frac{\rho}{\langle y\rangle^{4}} u, u\right\rangle_{L^{2}} \\
&+\int_{\mathbb{R}^{n}}\left(\sum_{j \notin I_{x}} \int_{\mathbb{R}^{n}} \frac{\left(1+|y|^{8}\right)\left(-\lambda_{j}(x)\right)\left[(Q(x) y)_{j}\right]^{2}}{\rho^{3}} \frac{y^{2}}{1+y^{2}} u^{2} d y\right) d x \\
& \leq \quad C\left\langle\frac{\left(1+|y|^{8}\right)\left\langle\partial_{x} V(x)\right\rangle^{4 / 3}|y|^{2}}{\rho^{3}} \frac{y^{2}}{1+y^{2}} u, u\right\rangle_{L^{2}}+C_{\sigma}\left(\|P u\|_{L^{2}}^{2}+\|u\|_{L^{2}}^{2}\right) \\
& \leq \quad \varepsilon\left\langle\frac{\left(1+|y|^{8}\right)\left\langle\partial_{x} V(x)\right\rangle^{2}}{\rho^{3}} \frac{y^{2}}{1+y^{2}} u, u\right\rangle_{L^{2}}+C_{\varepsilon}\left\langle\frac{\left(1+|y|^{8}\right)\langle y\rangle^{6}}{\rho^{3}} \frac{y^{2}}{1+y^{2}} u, u\right\rangle_{L^{2}} \\
&+C_{\sigma}\left(\|P u\|_{L^{2}}^{2}+\|u\|_{L^{2}}^{2}\right) \\
& \leq \varepsilon\left\langle\frac{\left(1+|y|^{8}\right)\left\langle\partial_{x} V(x)\right\rangle^{2}}{\rho^{3}} \frac{y^{2}}{1+y^{2}} u, u\right\rangle_{L^{2}}+C_{\varepsilon, \sigma}\left(\|P u\|_{L^{2}}^{2}+\|u\|_{L^{2}}^{2}\right),
\end{aligned}
$$

the second and last inequalities holding because

$$
\left\langle\partial_{x} V(x)\right\rangle^{4 / 3}|y|^{2} \leq \varepsilon\left\langle\partial_{x} V(x)\right\rangle^{2}+C_{\varepsilon}|y|^{6}
$$

and

$$
\left\langle\frac{\left(1+|y|^{8}\right)\langle y\rangle^{6}}{\rho^{3}} \frac{y^{2}}{1+y^{2}} u, u\right\rangle_{L^{2}} \leq C\|\langle y\rangle u\|_{L^{2}}^{2} \leq C\left(\|P u\|_{L^{2}}^{2}+\|u\|_{L^{2}}^{2}\right) .
$$

Now letting $\varepsilon=\frac{\sigma}{2}$, we obtain

$$
\begin{aligned}
& \frac{\sigma}{2}\left\langle\frac{\left(1+|y|^{8}\right)\left\langle\partial_{x} V(x)\right\rangle^{2}}{\rho^{3}} \frac{y^{2}}{1+y^{2}} u, u\right\rangle_{L^{2}}+\left\langle\frac{\rho}{\langle y\rangle^{4}} u, u\right\rangle_{L^{2}} \\
&+\int_{\mathbb{R}^{n}}\left(\sum_{j \notin I_{x}} \int_{\mathbb{R}^{n}} \frac{\left(1+|y|^{8}\right)\left(-\lambda_{j}(x)\right)\left[(Q(x) y)_{j}\right]^{2}}{\rho^{3}} \frac{y^{2}}{1+y^{2}} u^{2} d y\right) d x \\
& \leq C_{\sigma}\left(\|P u\|_{L^{2}}^{2}+\|u\|_{L^{2}}^{2}\right),
\end{aligned}
$$


and thus, choosing $\sigma=1 / 2$,

$$
\begin{gathered}
\left\langle\frac{\left(1+|y|^{8}\right)\left\langle\partial_{x} V(x)\right\rangle^{2}}{\rho^{3}} \frac{y^{2}}{1+y^{2}} u, u\right\rangle_{L^{2}}+\left\langle\frac{\rho}{\langle y\rangle^{4}} u, u\right\rangle_{L^{2}} \\
+\int_{\mathbb{R}^{n}}\left(\sum_{j \notin I_{x}} \int_{\mathbb{R}^{n}} \frac{\left(-\lambda_{j}(x)\right)\left[(Q(x) y)_{j}\right]^{2} y^{2}}{\left\langle\partial_{x} V(x)\right\rangle^{3}\langle y\rangle^{6}} u^{2} d y\right) d x \\
\leq C\left(\|P u\|_{L^{2}}^{2}+\|u\|_{L^{2}}^{2}\right)
\end{gathered}
$$

due to the fact that $-\lambda_{j}(x) \geq 0$ for $j \notin I_{x}$ and

$$
\frac{1}{\left\langle\partial_{x} V(x)\right\rangle^{3}\langle y\rangle^{6}} \leq C \frac{\left(1+|y|^{8}\right)}{\rho^{3}} \frac{1}{1+y^{2}} .
$$

In the following discussions we will give the lower bound of the summation on the left side of (14). To do so, we use the the estimates

$$
\begin{aligned}
\left\langle\partial_{x} V(x)\right\rangle^{\frac{1}{4}} \frac{y^{2}}{1+y^{2}} & \leq\left(\frac{1}{8} \frac{\left\langle\partial_{x} V(x)\right\rangle^{2}\langle y\rangle^{8}}{\rho^{3}}+\frac{3}{8} \frac{\rho}{\langle y\rangle^{4}}+\frac{\langle y\rangle}{2}\right) \frac{y^{2}}{1+y^{2}} \\
& \leq C \frac{\left\langle\partial_{x} V(x)\right\rangle^{2}\left(1+|y|^{8}\right)}{\rho^{3}} \frac{y^{2}}{1+y^{2}}+\frac{\rho}{\langle y\rangle^{4}}+\langle y\rangle,
\end{aligned}
$$

together with (14), to conclude

$$
\sum_{j=1}^{n}\left\|\left\langle\partial_{x} V(x)\right\rangle^{\frac{1}{8}} y_{j}\langle y\rangle^{-1} u\right\|_{L^{2}}^{2} \leq C\left(\|P u\|_{L^{2}}^{2}+\left\|\langle y\rangle^{1 / 2} u\right\|_{L^{2}}^{2}\right) \leq C\left(\|P u\|_{L^{2}}^{2}+\|u\|_{L^{2}}^{2}\right) .
$$

Moreover applying (9) with $v=\langle y\rangle^{-1} u$ and $\theta(x) \cdot y=\left\langle\partial_{x} V(x)\right\rangle^{\frac{1}{8}} y_{j}$, we get

$$
\begin{aligned}
\left\|\left\langle\partial_{x} V(x)\right\rangle^{\frac{1}{16}}\langle y\rangle^{-1} u\right\|_{L^{2}}^{2} & \leq C\left(\left\|\left\langle\partial_{x} V(x)\right\rangle^{\frac{1}{8}} y_{j}\langle y\rangle^{-1} u\right\|_{L^{2}}^{2}+\left\|\partial_{y_{j}}\langle y\rangle^{-1} u\right\|_{L^{2}}^{2}\right) \\
& \leq C\left(\left\|\left\langle\partial_{x} V(x)\right\rangle^{\frac{1}{8}} y_{j}\langle y\rangle^{-1} u\right\|_{L^{2}}^{2}+\left\|\partial_{y_{j}} u\right\|_{L^{2}}^{2}+\|u\|_{L^{2}}^{2}\right) \\
& \leq C\left(\|P u\|_{L^{2}}^{2}+\|u\|_{L^{2}}^{2}\right),
\end{aligned}
$$

the last inequality following from (15) and (8). As a result, observe

$$
\begin{aligned}
& \left\|\left\langle\partial_{x} V(x)\right\rangle^{\frac{1}{16}} u\right\|_{L^{2}}^{2} \\
= & \left(\left\langle\partial_{x} V(x)\right\rangle^{\frac{1}{16}} \frac{1}{1+|y|^{2}} u,\left\langle\partial_{x} V(x)\right\rangle^{\frac{1}{16}} u\right)_{L^{2}}+\sum_{j=1}^{n}\left(\left\langle\partial_{x} V(x)\right\rangle^{\frac{1}{16}} \frac{y_{j}^{2}}{1+|y|^{2}} u,\left\langle\partial_{x} V(x)\right\rangle^{\frac{1}{16}} u\right)_{L^{2}} \\
= & \left\|\left\langle\partial_{x} V(x)\right\rangle^{\frac{1}{16}}\langle y\rangle^{-1} u\right\|_{L^{2}}^{2}+\sum_{j=1}^{n}\left\|\left\langle\partial_{x} V(x)\right\rangle^{\frac{1}{16}} y_{j}\langle y\rangle^{-1} u\right\|_{L^{2}}^{2} \\
\leq & \left\|\left\langle\partial_{x} V(x)\right\rangle^{\frac{1}{16}}\langle y\rangle^{-1} u\right\|_{L^{2}}^{2}+\sum_{j=1}^{n}\left\|\left\langle\partial_{x} V(x)\right\rangle^{\frac{1}{8}} y_{j}\langle y\rangle^{-1} u\right\|_{L^{2}}^{2},
\end{aligned}
$$

and thus combining the above inequalities and (15), we obtain

$$
\left\|\left\langle\partial_{x} V(x)\right\rangle^{\frac{1}{16}} u\right\|_{L^{2}}^{2} \leq C\left(\|P u\|_{L^{2}}^{2}+\|u\|_{L^{2}}^{2}\right) .
$$

Then the conclusion (i) in Theorem 1.2 follows. 
Now we prove the conclusion (ii). Let $x \in \mathbb{R}^{n}$ be given and let $1 \leq i, \ell \leq n$ and $j \notin I_{x}$. Recall $Q(x)=\left(q_{k \ell}(x)\right)_{1 \leq k, \ell \leq n}$. Similarly as above, applying again (9) with

$v=y_{\ell}\langle y\rangle^{-3} u, \quad \theta(x) \cdot y=\left(-\lambda_{j}(x)\right)^{1 / 2}\left\langle\partial_{x} V(x)\right\rangle^{-\frac{3}{2}}(Q(x) y)_{j}=\left(-\lambda_{j}(x)\right)^{1 / 2}\left\langle\partial_{x} V(x)\right\rangle^{-\frac{3}{2}} \sum_{k=1}^{n} q_{j k} y_{k}$,

we have,

$$
\begin{aligned}
& \left\|\left(-\lambda_{j}(x)\right)^{1 / 4}\left\langle\partial_{x} V(x)\right\rangle^{-3 / 4}\left|q_{j i}(x)\right|^{1 / 2} y_{\ell}\langle y\rangle^{-3} u\right\|_{L^{2}\left(\mathbb{R}_{y}^{n}\right)}^{2} \\
\leq & C\left(\left\|\left(-\lambda_{j}(x)\right)^{1 / 2}\left\langle\partial_{x} V(x)\right\rangle^{-3 / 2}\left[(Q(x) y)_{j}\right] y_{\ell}\langle y\rangle^{-3} u\right\|_{L^{2}\left(\mathbb{R}_{y}^{n}\right)}^{2}+\left\|\partial_{y_{i}} y_{\ell}\langle y\rangle^{-3} u\right\|_{L^{2}\left(\mathbb{R}_{y}^{n}\right)}^{2}\right) \\
\leq & C \int_{\mathbb{R}^{n}} \frac{\left(-\lambda_{j}(x)\right)\left[(Q(x) y)_{j}\right]^{2} y^{2}}{\left\langle\partial_{x} V(x)\right\rangle^{3}\langle y\rangle^{6}} u^{2} d y+C\left(\left\|\partial_{y_{i}} u\right\|_{L^{2}\left(\mathbb{R}_{y}^{n}\right)}^{2}+\|u\|_{L^{2}\left(\mathbb{R}_{y}^{n}\right)}^{2}\right) .
\end{aligned}
$$

Thus, combining (14) and (8),

$$
\begin{aligned}
& \int_{\mathbb{R}^{n}}\left(\sum_{i=1}^{n} \sum_{j \notin I_{x}}\left\|\left(-\lambda_{j}(x)\right)^{1 / 4}\left\langle\partial_{x} V(x)\right\rangle^{-3 / 4}\left|q_{j i}(x)\right|^{1 / 2} y_{\ell}\langle y\rangle^{-3} u\right\|_{L^{2}\left(\mathbb{R}_{y}^{n}\right)}^{2}\right) d x \\
\leq & C \int_{\mathbb{R}^{n}}\left(\sum_{j \notin I_{x}} \int_{\mathbb{R}^{n}} \frac{\left(-\lambda_{j}(x)\right)\left[(Q(x) y)_{j}\right]^{2} y^{2}}{\left\langle\partial_{x} V(x)\right\rangle^{3}\langle y\rangle^{6}} u^{2} d y\right) d x+C \sum_{i=1}^{n}\left(\left\|\partial_{y_{i}} u\right\|_{L^{2}}^{2}+\|u\|_{L^{2}}^{2}\right) \\
\leq & C\left(\|P u\|_{L^{2}}^{2}+\|u\|_{L^{2}}^{2}\right) .
\end{aligned}
$$

Moreover, using again (9) with

$$
v=\langle y\rangle^{-3} u, \quad \theta \cdot y=\left(-\lambda_{j}(x)\right)^{1 / 4}\left\langle\partial_{x} V(x)\right\rangle^{-3 / 4}\left|q_{j i}(x)\right|^{1 / 2} y_{\ell},
$$

gives,

$$
\begin{aligned}
& \int_{\mathbb{R}^{n}}\left(\sum_{i=1}^{n} \sum_{j \notin I_{x}}\left\|\left(-\lambda_{j}(x)\right)^{1 / 8}\left\langle\partial_{x} V(x)\right\rangle^{-3 / 8}\left|q_{j i}(x)\right|^{1 / 4}\langle y\rangle^{-3} u\right\|_{L^{2}\left(\mathbb{R}_{y}^{n}\right)}\right) d x \\
\leq & \int_{\mathbb{R}^{n}}\left(\sum_{i=1}^{n} \sum_{j \notin I_{x}}\left\|\left(-\lambda_{j}(x)\right)^{1 / 4}\left\langle\partial_{x} V(x)\right\rangle^{-3 / 4}\left|q_{j i}(x)\right|^{1 / 2} y_{\ell}\langle y\rangle^{-3} u\right\|_{L^{2}\left(\mathbb{R}_{y}^{n}\right)}^{2}\right) d x+C\left\|\partial_{y_{\ell}}\langle y\rangle^{-3} u\right\|_{L^{2}}^{2} \\
\leq & C\left(\|P u\|_{L^{2}}^{2}+\|u\|_{L^{2}}^{2}\right) .
\end{aligned}
$$

the last inequality following from (17) and (8). On the other hand, in view of (12) we see

$$
\sum_{i=1}^{n} \sum_{j=1}^{n} \lambda_{j}(x)\left(q_{j i}(x)\right)^{2}=\sum_{j=1}^{n} \lambda_{j}(x)=\Delta_{x} V(x) .
$$

Then, by the assumption (5) in Theorem [1.2, we can find a constant $C_{\alpha}$ depending on $\alpha$, such that

$$
\forall x \in \mathbb{R}^{n}, \quad 0 \leq \alpha\left|\partial_{x} V(x)\right|^{2}-\Delta_{x} V(x)+C_{\alpha} \leq C_{\alpha}\left\langle\partial_{x} V(x)\right\rangle^{2}+\sum_{i=1}^{n} \sum_{j \notin I_{x}}^{n}\left(-\lambda_{j}(x)\right)\left|q_{j i}(x)\right|^{2},
$$

the last inequality following from (19). And thus for any $x \in \mathbb{R}^{n}$,

$$
\left.|\alpha| \partial_{x} V(x)\right|^{2}-\left.\Delta_{x} V(x)\right|^{1 / 4} \leq C\left\langle\partial_{x} V(x)\right\rangle^{1 / 2}+C \sum_{i=1}^{n} \sum_{j \notin I_{x}}^{n}\left(-\lambda_{j}(x)\right)^{1 / 4}\left|q_{j i}(x)\right|^{1 / 2},
$$


which, together with (18), yields

$$
\begin{aligned}
& \left\langle\frac{\left.|\alpha| \partial_{x} V(x)\right|^{2}-\left.\Delta_{x} V(x)\right|^{\frac{1}{4}}}{\left\langle\partial_{x} V(x)\right\rangle^{3 / 4}\langle y\rangle^{6}} u, u\right\rangle_{L^{2}} \\
\leq & C\left\langle\frac{\left\langle\partial_{x} V(x)\right\rangle^{1 / 2}}{\left\langle\partial_{x} V(x)\right\rangle^{3 / 4}\langle y\rangle^{6}} u, u\right\rangle_{L^{2}}+C \int_{\mathbb{R}^{n}}\left(\sum_{i=1}^{n} \sum_{j \notin I_{x}}\left\langle\frac{\left(-\lambda_{j}(x)\right)^{1 / 4}\left|q_{j i}(x)\right|^{1 / 2}}{\left\langle\partial_{x} V(x)\right\rangle^{3 / 4}\langle y\rangle^{6}} u, u\right\rangle_{L^{2}\left(\mathbb{R}_{y}^{n}\right)}\right) d x \\
\leq & C\left(\|P u\|_{L^{2}}^{2}+\|u\|_{L^{2}}^{2}\right) .
\end{aligned}
$$

As a result, we conclude, combining (16), (8) and the above inequality,

$$
\left\|\left.|\alpha| \partial_{x} V(x)\right|^{2}-\left.\Delta_{x} V(x)\right|^{1 / 80} u\right\|_{L^{2}}^{2} \leq C\left(\|P u\|_{L^{2}}^{2}+\|u\|_{L^{2}}^{2}\right),
$$

due to the estimate

$$
\left.|\alpha| \partial_{x} V(x)\right|^{2}-\left.\Delta_{x} V(x)\right|^{1 / 40} \leq \frac{1}{10} \frac{\left.|\alpha| \partial_{x} V(x)\right|^{2}-\left.\Delta_{x} V(x)\right|^{\frac{1}{4}}}{\left\langle\partial_{x} V(x)\right\rangle^{3 / 4}\langle y\rangle^{6}}+\frac{3}{5}\left\langle\partial_{x} V(x)\right\rangle^{1 / 8}+\frac{3}{10}\langle y\rangle^{2} .
$$

Thus the proof of Theorem 1.2 is complete.

2.2. Proof of Theorem 1.3. This subsection is devoted to proving Theorem 1.3. Similarly as Lemma 2.1 we have the following

Lemma 2.2. Let $V(x) \in C^{2}\left(\mathbb{R}^{n}\right)$. Then for all $\left.\sigma \in\right] 0,1\left[\right.$ there exists a constant $C_{\sigma}>0$ such that for any $u \in C_{0}^{\infty}\left(\mathbb{R}^{2 n}\right)$ we have

$$
\begin{aligned}
& \sigma\left\langle\frac{\left\langle\partial_{x} V(x)\right\rangle^{2}}{\left\langle\partial_{x} V(x) \cdot y\right\rangle^{3}} \frac{y^{2}}{1+y^{2}} u, u\right\rangle_{L^{2}}+\left\langle\frac{2\left\langle\partial_{x} V(x) \cdot y\right\rangle}{\langle y\rangle^{4}} u, u\right\rangle_{L^{2}}-\sum_{1 \leq i, j \leq n}\left\langle\frac{\left(\partial_{x_{i} x_{j}} V\right) y_{i} y_{j}}{\left\langle\partial_{x} V(x) \cdot y\right\rangle^{3}} \frac{y^{2}}{1+y^{2}} u, u\right\rangle_{L^{2}} \\
& \leq C_{\sigma}\left(\|P u\|_{L^{2}}^{2}+\|u\|_{L^{2}}^{2}\right) .
\end{aligned}
$$

Proof. The proof is quite similar as Lemma 2.1. Let $\mathcal{K} \in C^{1}\left(\mathbb{R}^{2 n}\right)$ be defined by

$$
\mathcal{K}=\mathcal{K}(x, y)=\frac{2 \partial_{x} V(x) \cdot y}{\left\langle\partial_{x} V(x) \cdot y\right\rangle} \frac{y^{2}}{1+y^{2}} .
$$

Then using the relation

$$
\frac{1}{2}\left[\mathcal{K}, X_{0}\right]=\frac{\partial_{x} V(x) \cdot y}{\left\langle\partial_{x} V(x) \cdot y\right\rangle}\left[\frac{y^{2}}{\left(1+y^{2}\right)}, X_{0}\right]+\left[\frac{\partial_{x} V(x) \cdot y}{\left\langle\partial_{x} V(x) \cdot y\right\rangle}, X_{0}\right] \frac{y^{2}}{\left(1+y^{2}\right)},
$$

we obtain

$$
\frac{1}{2}\left[\mathcal{K}, X_{0}\right]=\frac{2\left|\partial_{x} V(x) \cdot y\right|^{2}}{\left\langle\partial_{x} V(x) \cdot y\right\rangle\left(1+y^{2}\right)^{2}}+\left(\frac{\left|\partial_{x} V(x)\right|^{2}}{\left\langle\partial_{x} V(x) \cdot y\right\rangle^{3}}-\sum_{1 \leq i, j \leq n} \frac{\left(\partial_{x_{i} x_{j}} V\right) y_{i} y_{j}}{\left\langle\partial_{x} V(x) \cdot y\right\rangle^{3}}\right) \frac{y^{2}}{1+y^{2}} .
$$

Thus using the relationship

$$
\operatorname{Re}\left\langle X_{0} u, \mathcal{K} u\right\rangle_{L^{2}}=\frac{1}{2}\left\langle\left[\mathcal{K}, X_{0}\right] u, u\right\rangle_{L^{2}},
$$


we conclude

$$
\begin{gathered}
\left\langle\frac{\left\langle\partial_{x} V(x)\right\rangle^{2}}{\left\langle\partial_{x} V(x) \cdot y\right\rangle^{3}} \frac{y^{2}}{1+y^{2}} u, u\right\rangle_{L^{2}}+\left\langle\frac{2\left\langle\partial_{x} V(x) \cdot y\right\rangle}{\left(1+y^{2}\right)^{2}} u, u\right\rangle_{L^{2}} \\
-\sum_{1 \leq i, j \leq n}\left\langle\frac{\left(\partial_{x_{i} x_{j}} V\right) y_{i} y_{j}}{\left\langle\partial_{x} V(x) \cdot y\right\rangle^{3}} \frac{y^{2}}{1+y^{2}} u, u\right\rangle_{L^{2}} \\
\leq \operatorname{Re}\left\langle X_{0} u, \mathcal{K} u\right\rangle_{L^{2}}+3\|u\|_{L^{2}}^{2} .
\end{gathered}
$$

On the other hand, since $\mathcal{K} \in L^{\infty}\left(\mathbb{R}^{2 n}\right)$ with $\|\mathcal{K}\|_{L^{\infty}} \leq 2$ then it is easy to see

$$
\left|\operatorname{Re}\langle P u, \mathcal{K} u\rangle_{L^{2}}\right| \leq\|P u\|_{L^{2}}^{2}+\|u\|_{L^{2}}^{2} \text {. }
$$

Recall $X_{j}=\partial_{y_{j}}+\frac{y_{j}}{2}, j=1, \cdots n$. Then direct computation gives, for each $1 \leq j \leq n$,

$$
\left[\mathcal{K}, X_{j}\right]=-\frac{2 \partial_{x_{j}} V(x)}{\left\langle\partial_{x} V(x) \cdot y\right\rangle^{3}} \frac{y^{2}}{1+y^{2}}-\frac{4\left(\partial_{x} V(x) \cdot y\right) y_{j}}{\left\langle\partial_{x} V(x) \cdot y\right\rangle\left(1+y^{2}\right)^{2}} .
$$

As a result, for any $\varepsilon>0$,

$$
\begin{aligned}
& \left|\operatorname{Re}\left\langle X_{j} u,\left[\mathcal{K}, X_{j}\right] u\right\rangle_{L^{2}}\right| \\
\leq & \varepsilon\left\|\left\langle\partial_{x} V(x) \cdot y\right\rangle^{-3}\left(1+y^{2}\right)^{-1} y^{2}\left|\partial_{x_{j}} V(x)\right| u\right\|_{L^{2}}^{2}+C_{\varepsilon}\left(\left\|X_{j} u\right\|_{L^{2}}^{2}+\|u\|_{L^{2}}^{2}\right) \\
\leq & \varepsilon\left\langle\frac{\left|\partial_{x_{j}} V(x)\right|^{2}}{\left\langle\partial_{x} V(x) \cdot y\right\rangle^{3}} \frac{y^{2}}{1+y^{2}} u, u\right\rangle_{L^{2}}+C_{\varepsilon}\left(\left\|X_{j} u\right\|_{L^{2}}^{2}+\|u\|_{L^{2}}^{2}\right) .
\end{aligned}
$$

This yields, using again the facts that $\|\mathcal{K}\|_{L^{\infty}} \leq 2$ and (8),

$$
\begin{aligned}
\sum_{j=1}^{n}\left|\operatorname{Re}\left\langle X_{j}^{*} X_{j} u, \mathcal{K} u\right\rangle_{L^{2}}\right| & \leq \sum_{j=1}^{n}\left(\left|\operatorname{Re}\left\langle X_{j} u, \mathcal{K} X_{j} u\right\rangle_{L^{2}}\right|+\left|\operatorname{Re}\left\langle X_{j} u,\left[\mathcal{K}, X_{j}\right] u\right\rangle_{L^{2}}\right|\right) \\
& \leq \varepsilon\left\langle\frac{\left|\partial_{x} V(x)\right|^{2}}{\left\langle\partial_{x} V(x) \cdot y\right\rangle^{3}} \frac{y^{2}}{1+y^{2}} u, u\right\rangle_{L^{2}}+C_{\varepsilon}\left(\|P u\|_{L^{2}}^{2}+\|u\|_{L^{2}}^{2}\right),
\end{aligned}
$$

which, along with (20), (21) and the relationship that

$$
\operatorname{Re}\left\langle X_{0} u, \mathcal{K} u\right\rangle_{L^{2}}=\operatorname{Re}\langle P u, \mathcal{K} u\rangle_{L^{2}}-\operatorname{Re} \sum_{j=1}^{n}\left\langle X_{j}^{*} X_{j} u, \mathcal{K} u\right\rangle_{L^{2}},
$$

implies

$$
\begin{gathered}
\left\langle\frac{\left\langle\partial_{x} V(x)\right\rangle^{2}}{\left\langle\partial_{x} V(x) \cdot y\right\rangle^{3}} \frac{y^{2}}{1+y^{2}} u, u\right\rangle_{L^{2}}+\left\langle\frac{2\left\langle\partial_{x} V(x) \cdot y\right\rangle}{\left(1+y^{2}\right)^{2}} u, u\right\rangle_{L^{2}} \\
-\sum_{1 \leq i, j \leq n}\left\langle\frac{\left(\partial_{x_{i} x_{j}} V\right) y_{i} y_{j}}{\left\langle\partial_{x} V(x) \cdot y\right\rangle^{3}} \frac{y^{2}}{1+y^{2}} u, u\right\rangle_{L^{2}} \\
\leq \varepsilon\left\langle\frac{\left|\partial_{x} V(x)\right|^{2}}{\left\langle\partial_{x} V(x) \cdot y\right\rangle^{3}} \frac{y^{2}}{1+y^{2}} u, u\right\rangle_{L^{2}}+C_{\varepsilon}\left(\|P u\|_{L^{2}}^{2}+\|u\|_{L^{2}}^{2}\right) .
\end{gathered}
$$

Given any $\sigma \in] 0,1[$, letting $\varepsilon=1-\sigma$ gives the desired estimate in Lemma 2.2. The proof is thus complete. 
Lemma 2.3. Let $\tau \geq 0$ be given. Then for any $\varepsilon>0$,

$$
\begin{aligned}
& \sum_{1 \leq i, j \leq n} \tau\left\langle\frac{\left\langle\partial_{x} V(x)\right\rangle^{4 / 5}\left(\partial_{x_{i}} V(x)\right)\left(\partial_{x_{j}} V(x)\right) y_{i} y_{j}}{\left\langle\partial_{x} V(x) \cdot y\right\rangle^{3}} \frac{y^{2}}{1+y^{2}} u, u\right\rangle_{L^{2}} \\
\leq & \varepsilon\left\langle\frac{\left\langle\partial_{x} V(x)\right\rangle^{2}}{\left\langle\partial_{x} V(x) \cdot y\right\rangle^{3}} \frac{y^{2}}{1+y^{2}} u, u\right\rangle_{L^{2}}+\varepsilon\left\langle\frac{\left\langle\partial_{x} V(x) \cdot y\right\rangle}{\langle y\rangle^{4}} u, u\right\rangle_{L^{2}}+C_{\varepsilon, \tau}\left(\|P u\|_{L^{2}}^{2}+\|u\|_{L^{2}}^{2}\right),
\end{aligned}
$$

where $C_{\varepsilon, \tau}$ is a constant depending only on $\varepsilon$ and $\tau$.

Proof. In the proof we use $C_{\varepsilon, \tau}$ to denote the different constants depending on $\varepsilon$ and $\tau$. Direct calculation gives

$$
\begin{aligned}
\tau \frac{\left\langle\partial_{x} V\right\rangle^{4 / 5}\left|\partial_{x} V(x) \cdot y\right|^{2}}{\left\langle\partial_{x} V(x) \cdot y\right\rangle^{3}} \frac{|y|^{2}}{1+|y|^{2}} & \leq \frac{\varepsilon\left\langle\partial_{x} V(x)\right\rangle^{2}+C_{\varepsilon, \tau}\left|\partial_{x} V(x) \cdot y\right|^{10 / 3}}{\left\langle\partial_{x} V(x) \cdot y\right\rangle^{3}} \frac{|y|^{2}}{1+|y|^{2}} \\
& \leq \varepsilon \frac{\left\langle\partial_{x} V(x)\right\rangle^{2}}{\left\langle\partial_{x} V(x) \cdot y\right\rangle^{3}} \frac{|y|^{2}}{1+|y|^{2}}+C_{\varepsilon, \tau}\left\langle\partial_{x} V(x) \cdot y\right\rangle^{1 / 3} \\
& \leq \varepsilon \frac{\left\langle\partial_{x} V(x)\right\rangle^{2}}{\left\langle\partial_{x} V(x) \cdot y\right\rangle^{3}} \frac{|y|^{2}}{1+|y|^{2}}+\varepsilon \frac{\left\langle\partial_{x} V(x) \cdot y\right\rangle}{\langle y\rangle^{4}}+C_{\varepsilon, \tau}\langle y\rangle^{2},
\end{aligned}
$$

and thus, using (8),

$$
\begin{aligned}
& \tau\left\langle\frac{\left\langle\partial_{x} V(x)\right\rangle^{4 / 5}\left|\partial_{x} V(x) \cdot y\right|^{2}}{\left\langle\partial_{x} V(x) \cdot y\right\rangle^{3}} \frac{y^{2}}{1+y^{2}} u, u\right\rangle_{L^{2}} \\
\leq & \varepsilon\left\langle\frac{\left\langle\partial_{x} V(x)\right\rangle^{2}}{\left\langle\partial_{x} V(x) \cdot y\right\rangle^{3}} \frac{y^{2}}{1+y^{2}} u, u\right\rangle_{L^{2}}+\varepsilon\left\langle\frac{\left\langle\partial_{x} V(x) \cdot y\right\rangle}{\langle y\rangle^{4}} u, u\right\rangle_{L^{2}}+C_{\varepsilon, \tau}\left(\|P u\|_{L^{2}}^{2}+\|u\|_{L^{2}}^{2}\right) .
\end{aligned}
$$

Then observing

$$
\sum_{1 \leq i, j \leq n} \tau\left\langle\frac{\left\langle\partial_{x} V\right\rangle^{4 / 5}\left(\partial_{x_{i}} V\right)\left(\partial_{x_{j}} V\right) y_{i} y_{j}}{\rho^{3}} \frac{y^{2}}{1+y^{2}} u, u\right\rangle_{L^{2}}=\tau\left\langle\frac{\left\langle\partial_{x} V\right\rangle^{4 / 5}\left|\partial_{x} V \cdot y\right|^{2}}{\rho^{3}} \frac{y^{2}}{1+y^{2}} u, u\right\rangle_{L^{2}},
$$

the desired estimate in Lemma 2.3 follows. The proof is complete.

The rest is occupied by the proof of Theorem 1.3 .

Proof of Theorem 1.3. By virtue of Lemma 2.2 and Lemma 2.3, we obtain, for all $0<\sigma<1$ and for any $\varepsilon>0$,

$$
\begin{aligned}
\sigma & \left\langle\frac{\left\langle\partial_{x} V(x)\right\rangle^{2}}{\left\langle\partial_{x} V(x) \cdot y\right\rangle^{3}} \frac{y^{2}}{1+y^{2}} u, u\right\rangle_{L^{2}}+\left\langle\frac{2\left\langle\partial_{x} V(x) \cdot y\right\rangle}{\langle y\rangle^{4}} u, u\right\rangle_{L^{2}} \\
& +\sum_{1 \leq i, j \leq n}\left\{\tau\left\langle\frac{\left\langle\partial_{x} V\right\rangle^{4 / 5}\left(\partial_{x_{i}} V\right)\left(\partial_{x_{j}} V\right) y_{i} y_{j}}{\left\langle\partial_{x} V \cdot y\right\rangle^{3}} \frac{y^{2}}{1+y^{2}} u, u\right\rangle_{L^{2}}-\left\langle\frac{\left(\partial_{x_{i} x_{j}} V\right) y_{i} y_{j}}{\left\langle\partial_{x} V(x) \cdot y\right\rangle^{3}} \frac{y^{2}}{1+y^{2}} u, u\right\rangle_{L^{2}}\right\} \\
\leq & \varepsilon\left\langle\frac{\left\langle\partial_{x} V(x)\right\rangle^{2}}{\left\langle\partial_{x} V(x) \cdot y\right\rangle^{3}} \frac{y^{2}}{1+y^{2}} u, u\right\rangle_{L^{2}}+\varepsilon\left\langle\frac{\left\langle\partial_{x} V(x) \cdot y\right\rangle}{\langle y\rangle^{4}} u, u\right\rangle_{L^{2}}+C_{\varepsilon, \tau, \sigma}\left(\|P u\|_{L^{2}}^{2}+\|u\|_{L^{2}}^{2}\right) .
\end{aligned}
$$

Letting $\varepsilon=\sigma / 2$, denoting by $C$ the different constants which may depend on $\tau$ and $\sigma$, we have

$$
\begin{aligned}
& \frac{\sigma}{2}\left\langle\frac{\left\langle\partial_{x} V(x)\right\rangle^{2}}{\left\langle\partial_{x} V(x) \cdot y\right\rangle^{3}} \frac{y^{2}}{1+y^{2}} u, u\right\rangle_{L^{2}}+\left\langle\frac{\left\langle\partial_{x} V(x) \cdot y\right\rangle}{\langle y\rangle^{4}} u, u\right\rangle_{L^{2}} \\
& \quad+\sum_{1 \leq i, j \leq n}\left\{\tau\left\langle\frac{\left\langle\partial_{x} V\right\rangle^{4 / 5}\left(\partial_{x_{i}} V\right)\left(\partial_{x_{j}} V\right) y_{i} y_{j}}{\left\langle\partial_{x} V \cdot y\right\rangle^{3}} \frac{y^{2}}{1+y^{2}} u, u\right\rangle_{L^{2}}-\left\langle\frac{\left(\partial_{x_{i} x_{j}} V\right) y_{i} y_{j}}{\left\langle\partial_{x} V(x) \cdot y\right\rangle^{3}} \frac{y^{2}}{1+y^{2}} u, u\right\rangle_{L^{2}}\right\} \\
& \leq C\left(\|P u\|_{L^{2}}^{2}+\|u\|_{L^{2}}^{2}\right),
\end{aligned}
$$


and thus, using (8),

$$
\begin{aligned}
& \frac{\sigma}{2}\left\langle\frac{\left\langle\partial_{x} V(x)\right\rangle^{2}}{\left\langle\partial_{x} V(x) \cdot y\right\rangle^{3}} \frac{y^{2}}{1+y^{2}} u, u\right\rangle_{L^{2}}+\left\langle\frac{\left\langle\partial_{x} V(x) \cdot y\right\rangle}{\langle y\rangle^{4}} u, u\right\rangle_{L^{2}}+\left\langle\frac{y^{T} A_{\tau}(x) y}{\left\langle\partial_{x} V \cdot y\right\rangle^{3}} \frac{y^{2}}{1+y^{2}} u, u\right\rangle_{L^{2}} \\
& \leq C\left(\|P u\|_{L^{2}}^{2}+\|u\|_{L^{2}}^{2}\right),
\end{aligned}
$$

where $A_{\tau}(x)$ is the matrix defined in Theorem 1.3, i.e.,

$$
A_{\tau}(x)=\left(a_{i j}^{\tau}(x)\right)_{1 \leq i, j \leq n}, \quad a_{i j}^{\tau}(x)=\tau\left\langle\partial_{x} V(x)\right\rangle^{\frac{4}{5}}\left(\partial_{x_{i}} V(x)\right)\left(\partial_{x_{j}} V(x)\right)-\partial_{x_{i} x_{j}} V(x)+\tau \delta_{i j} .
$$

Now under the assumption that $A_{\tau}(x)$ is positive-definite, we can find its Cholesky decomposition matrix

$$
B_{\tau}(x)=\left(b_{i j}^{\tau}(x)\right)_{1 \leq i, j \leq n},
$$

satisfying the relation

$$
A_{\tau}=B_{\tau}^{T} B_{\tau}
$$

Then using the following estimates

$$
\begin{aligned}
& \sum_{\ell=1}^{n} \sum_{j=1}^{n}\left\|\sum_{1 \leq k \leq n} b_{j k}^{\tau}(x)\left\langle\partial_{x} V(x)\right\rangle^{-3 / 2} y_{k} y_{\ell}\langle y\rangle^{-5 / 2} u\right\|_{L^{2}}^{2} \\
= & \left\langle\frac{B_{\tau}(x) y}{\left\langle\partial_{x} V(x)\right\rangle^{3}\langle y\rangle^{3}} \frac{y^{2}}{1+y^{2}} u, B_{\tau}(x) y u\right\rangle_{L^{2}} \\
\leq & C\left\langle\frac{B_{\tau}(x) y}{\left\langle\partial_{x} V(x) \cdot y\right\rangle^{3}} \frac{y^{2}}{1+y^{2}} u, B_{\tau}(x) y u\right\rangle_{L^{2}}=C\left\langle\frac{y^{T} A_{\tau}(x) y}{\left\langle\partial_{x} V(x) \cdot y\right\rangle^{3}} \frac{y^{2}}{1+y^{2}} u, u\right\rangle_{L^{2}}
\end{aligned}
$$

and (22), we have, letting $\sigma=1 / 2$,

$$
\begin{aligned}
& \left\langle\frac{\left\langle\partial_{x} V(x)\right\rangle^{2}}{\left\langle\partial_{x} V(x) \cdot y\right\rangle^{3}} \frac{y^{2}}{1+y^{2}} u, u\right\rangle_{L^{2}}+\left\langle\frac{\left\langle\partial_{x} V(x) \cdot y\right\rangle}{\langle y\rangle^{4}} u, u\right\rangle_{L^{2}} \\
& \quad+\sum_{\ell=1}^{n} \sum_{j=1}^{n}\left\|\sum_{1 \leq k \leq n} b_{j k}^{\tau}(x)\left\langle\partial_{x} V(x)\right\rangle^{-3 / 2} y_{k} y_{\ell}\langle y\rangle^{-5 / 2} u\right\|_{L^{2}}^{2} \\
& \leq C\left(\|P u\|_{L^{2}}^{2}+\|u\|_{L^{2}}^{2}\right) .
\end{aligned}
$$

Moreover observe

$$
\begin{aligned}
& \sum_{\ell=1}^{n}\left\|\left\langle\partial_{x} V(x)\right\rangle^{\frac{1}{10}} y_{\ell}\langle y\rangle^{-1} u\right\|_{L^{2}}^{2} \\
\leq & \left\langle\frac{\left|\partial_{x} V(x)\right|^{2}}{\left\langle\partial_{x} V(x) \cdot y\right\rangle^{3}} \frac{y^{2}}{1+y^{2}} u, u\right\rangle_{L^{2}}+\left\langle\frac{\left\langle\partial_{x} V(x) \cdot y\right\rangle}{\langle y\rangle^{4}} u, u\right\rangle_{L^{2}}+\|\langle y\rangle u\|_{L^{2}}^{2},
\end{aligned}
$$

due to the estimate

$$
\begin{aligned}
\left\langle\partial_{x} V(x)\right\rangle^{\frac{1}{5}} \frac{y^{2}}{1+y^{2}} & \leq\left(\frac{1}{10} \frac{\left\langle\partial_{x} V(x)\right\rangle^{2}}{\left\langle\partial_{x} V(x) \cdot y\right\rangle^{3}}+\frac{9}{10}\left\langle\partial_{x} V(x) \cdot y\right\rangle^{1 / 3}\right) \frac{y^{2}}{1+y^{2}} \\
& \leq \frac{\left\langle\partial_{x} V(x)\right\rangle^{2}}{\left\langle\partial_{x} V(x) \cdot y\right\rangle^{3}} \frac{y^{2}}{1+y^{2}}+\frac{\left\langle\partial_{x} V(x) \cdot y\right\rangle}{\langle y\rangle^{4}}+\langle y\rangle^{2}
\end{aligned}
$$


Then combining the above inequalities, (8) and (23), we have

$$
\begin{aligned}
& \sum_{\ell=1}^{n}\left\|\left\langle\partial_{x} V(x)\right\rangle^{\frac{1}{10}} y_{\ell}\langle y\rangle^{-1} u\right\|_{L^{2}}^{2} \\
& +\sum_{\ell=1}^{n} \sum_{j=1}^{n}\left\|\sum_{1 \leq k \leq n} b_{j k}^{\tau}(x)\left\langle\partial_{x} V(x)\right\rangle^{-3 / 2} y_{k} y_{\ell}\langle y\rangle^{-5 / 2} u\right\|_{L^{2}}^{2} \\
& \leq C\left(\|P u\|_{L^{2}}^{2}+\|u\|_{L^{2}}^{2}\right) .
\end{aligned}
$$

In order to obtain a lower bound of the terms on the left hand side of (24), we will use (9) with

$$
\theta \cdot y=\sum_{\ell=1}^{n}\left\langle\partial_{x} V(x)\right\rangle^{\frac{1}{10}} y_{\ell}, \quad v=\langle y\rangle^{-1} u
$$

this implies

$$
\begin{aligned}
\left\|\left\langle\partial_{x} V(x)\right\rangle^{\frac{1}{20}}\langle y\rangle^{-1} u\right\|_{L^{2}} & \leq C\left(\left\|\sum_{\ell=1}^{n}\left\langle\partial_{x} V(x)\right\rangle^{\frac{1}{10}} y_{\ell}\langle y\rangle^{-1} u\right\|_{L^{2}}+\left\|\partial_{y_{k}}\langle y\rangle^{-1} u\right\|_{L^{2}}\right) \\
& \leq C \sum_{\ell=1}^{n}\left\|\left\langle\partial_{x} V(x)\right\rangle^{\frac{1}{10}} y_{\ell}\langle y\rangle^{-1} u\right\|_{L^{2}}+C\left(\|P u\|_{L^{2}}+\|u\|_{L^{2}}\right) .
\end{aligned}
$$

As a result, it follows from the above inequalities and (24) that

$$
\begin{aligned}
& \left\|\left\langle\partial_{x} V(x)\right\rangle^{\frac{1}{20}}\langle y\rangle^{-1} u\right\|_{L^{2}}^{2}+\sum_{\ell=1}^{n} \sum_{j=1}^{n}\left\|\sum_{1 \leq k \leq n} b_{j k}^{\tau}(x)\left\langle\partial_{x} V(x)\right\rangle^{-3 / 2} y_{k} y_{\ell}\langle y\rangle^{-5 / 2} u\right\|_{L^{2}}^{2} \\
\leq & C\left(\|P u\|_{L^{2}}^{2}+\|u\|_{L^{2}}^{2}\right),
\end{aligned}
$$

and thus, using again (24) and repeating the arguments used to prove (16),

$$
\begin{aligned}
& \left\|\left\langle\partial_{x} V(x)\right\rangle^{\frac{1}{20}} u\right\|_{L^{2}}^{2}+\sum_{\ell=1}^{n} \sum_{j=1}^{n}\left\|\sum_{1 \leq k \leq n} b_{j k}^{\tau}(x)\left\langle\partial_{x} V(x)\right\rangle^{-3 / 2} y_{k} y_{\ell}\langle y\rangle^{-5 / 2} u\right\|_{L^{2}}^{2} \\
\leq & C\left(\|P u\|_{L^{2}}^{2}+\|u\|_{L^{2}}^{2}\right) .
\end{aligned}
$$

Similarly, for any $1 \leq j, \ell \leq n$ we use (9) again with

$$
\theta \cdot y=\sum_{k=1}^{n} b_{j k}^{\tau}(x)\left\langle\partial_{x} V(x)\right\rangle^{-3 / 2} y_{k}, \quad v=y_{\ell}\langle y\rangle^{-5 / 2} u
$$

to obtain

$$
\begin{aligned}
& \sum_{1 \leq p \leq n}\left\|\left|b_{j p}^{\tau}(x)\right|^{1 / 2}\left\langle\partial_{x} V(x)\right\rangle^{-3 / 4} y_{\ell}\langle y\rangle^{-5 / 2} u\right\|_{L^{2}} \\
\leq & C \sum_{1 \leq p \leq n}\left(\left\|\sum_{1 \leq k \leq n} b_{j k}^{\tau}(x)\left\langle\partial_{x} V(x)\right\rangle^{-3 / 2} y_{k} y_{\ell}\langle y\rangle^{-5 / 2} u\right\|_{L^{2}}+\left\|\partial_{y_{p}} y_{\ell}\langle y\rangle^{-5 / 2} u\right\|_{L^{2}}\right) \\
\leq & C\left\|\sum_{1 \leq k \leq n} b_{j k}^{\tau}(x)\left\langle\partial_{x} V(x)\right\rangle^{-3 / 2} y_{k} y_{\ell}\langle y\rangle^{-5 / 2} u\right\|_{L^{2}}+C \sum_{1 \leq p \leq n}\left(\left\|\partial_{y_{p}} u\right\|_{L^{2}}+\|u\|_{L^{2}}\right) \\
\leq & C\left\|\sum_{1 \leq k \leq n} b_{j k}^{\tau}(x)\left\langle\partial_{x} V(x)\right\rangle^{-3 / 2} y_{k} y_{\ell}\langle y\rangle^{-5 / 2} u\right\|_{L^{2}}+C\left(\|P u\|_{L^{2}}+\|u\|_{L^{2}}\right) .
\end{aligned}
$$


This, along with (25) and (24), yields

$$
\begin{gathered}
\left\|\left\langle\partial_{x} V(x)\right\rangle^{\frac{1}{20}} u\right\|_{L^{2}}+\sum_{\ell=1}^{n}\left\|\left\langle\partial_{x} V(x)\right\rangle^{\frac{1}{10}} y_{\ell}\langle y\rangle^{-1} u\right\|_{L^{2}} \\
+\sum_{\ell=1}^{n} \sum_{j=1}^{n} \sum_{p=1}^{n}\left\|\left|b_{j p}^{\tau}(x)\right|^{1 / 2}\left\langle\partial_{x} V(x)\right\rangle^{-3 / 4} y_{\ell}\langle y\rangle^{-5 / 2} u\right\|_{L^{2}} \\
\leq C\left(\|P u\|_{L^{2}}+\|u\|_{L^{2}}\right) .
\end{gathered}
$$

Moreover, observing

$$
\left|b_{j p}^{\tau}(x)\right|^{1 / 17}\langle y\rangle^{45 / 34} \leq \frac{2}{17}\left|b_{j p}^{\tau}(x)\right|^{1 / 2}\left\langle\partial_{x} V(x)\right\rangle^{-3 / 4}+\frac{15}{17}\left\langle\partial_{x} V(x)\right\rangle^{1 / 10}\langle y\rangle^{3 / 2}
$$

due to Young's inequality, we conclude

$$
\begin{aligned}
& \left\|\left|b_{j p}^{\tau}(x)\right|^{1 / 17}\langle y\rangle^{45 / 34} y_{\ell}\langle y\rangle^{-5 / 2} u\right\|_{L^{2}} \\
\leq & C\left\|\left|b_{j p}^{\tau}(x)\right|^{1 / 2}\left\langle\partial_{x} V(x)\right\rangle^{-3 / 4} y_{\ell}\langle y\rangle^{-5 / 2} u\right\|_{L^{2}}+C\left\|\left\langle\partial_{x} V(x)\right\rangle^{\frac{1}{10}}\langle y\rangle^{3 / 2} y_{\ell}\langle y\rangle^{-5 / 2} u\right\|_{L^{2}},
\end{aligned}
$$

that is,

$$
\begin{aligned}
& \left\|\left|b_{j p}^{\tau}(x)\right|^{\frac{1}{17}} y_{\ell}\langle y\rangle^{-\frac{20}{17}} u\right\|_{L^{2}} \\
\leq & C\left\|\left|b_{j p}^{\tau}(x)\right|^{1 / 2}\left\langle\partial_{x} V(x)\right\rangle^{-3 / 4} y_{\ell}\langle y\rangle^{-5 / 2} u\right\|_{L^{2}}+C\left\|\left\langle\partial_{x} V(x)\right\rangle^{\frac{1}{10}} y_{\ell}\langle y\rangle^{-1} u\right\|_{L^{2}} .
\end{aligned}
$$

Combing the above estimate and (26), it follows that

$$
\left\|\left\langle\partial_{x} V(x)\right\rangle^{\frac{1}{20}} u\right\|_{L^{2}}+\sum_{\ell=1}^{n} \sum_{j=1}^{n} \sum_{p=1}^{n}\left\|\left|b_{j p}^{\tau}(x)\right|^{\frac{1}{17}} y_{\ell}\langle y\rangle^{-\frac{20}{17}} u\right\|_{L^{2}} \leq C\left(\|P u\|_{L^{2}}+\|u\|_{L^{2}}\right) .
$$

Now we use (9) to obtain

$$
\begin{aligned}
\left\|\left|b_{j p}^{\tau}(x)\right|^{\frac{1}{34}}\langle y\rangle^{-\frac{20}{17}} u\right\|_{L^{2}} & \leq C\left(\left\|\left|b_{j p}^{\tau}(x)\right|^{\frac{1}{17}} y_{\ell}\langle y\rangle^{-\frac{20}{17}} u\right\|_{L^{2}}+\left\|\partial_{y_{\ell}}\langle y\rangle^{-\frac{20}{17}} u\right\|_{L^{2}}\right) \\
& \leq C\left\|\mid b_{j p}^{\tau}(x)^{\frac{1}{17}} y_{\ell}\langle y\rangle^{-\frac{20}{17}} u\right\|_{L^{2}}+C\left(\|P u\|_{L^{2}}+\|u\|_{L^{2}}\right),
\end{aligned}
$$

which together with (27) gives

$$
\begin{aligned}
& \left\|\left\langle\partial_{x} V(x)\right\rangle^{\frac{1}{20}} u\right\|_{L^{2}}+\sum_{\ell=1}^{n} \sum_{j=1}^{n} \sum_{p=1}^{n}\left\|\left|b_{j p}^{\tau}(x)\right|^{\frac{1}{17}} y_{\ell}\langle y\rangle^{-\frac{20}{17}} u\right\|_{L^{2}}+\sum_{j=1}^{n} \sum_{p=1}^{n}\left\|\left|b_{j p}^{\tau}(x)\right|^{\frac{1}{34}}\langle y\rangle^{-\frac{20}{17}} u\right\|_{L^{2}} \\
\leq & C\left(\|P u\|_{L^{2}}+\|u\|_{L^{2}}\right),
\end{aligned}
$$

that is,

$$
\left\|\left\langle\partial_{x} V(x)\right\rangle^{\frac{1}{20}} u\right\|_{L^{2}}+\sum_{j=1}^{n} \sum_{p=1}^{n}\left\|\left|b_{j p}^{\tau}(x)\right|^{\frac{1}{34}}\langle y\rangle^{-\frac{3}{17}} u\right\|_{L^{2}} \leq C\left(\|P u\|_{L^{2}}+\|u\|_{L^{2}}\right) .
$$

As a result, using the inequality

we conclude

$$
\left|b_{j p}^{\tau}(x)\right|^{\frac{1}{40}} \leq \frac{17}{20}\left|b_{j p}^{\tau}(x)\right|^{\frac{1}{34}}\langle y\rangle^{-\frac{3}{17}}+\frac{3}{20}\langle y\rangle,
$$

$$
\begin{aligned}
\left\|\left\langle\partial_{x} V(x)\right\rangle^{\frac{1}{20}} u\right\|_{L^{2}}+\sum_{j=1}^{n} \sum_{p=1}^{n}\left\|\left|b_{j p}^{\tau}(x)\right|^{\frac{1}{40}} u\right\|_{L^{2}} & \leq C\left(\|P u\|_{L^{2}}+\|\langle y\rangle u\|_{L^{2}}\right) . \\
& \leq C\left(\|P u\|_{L^{2}}+\|u\|_{L^{2}}\right) .
\end{aligned}
$$


Recall $A_{\tau}=B_{\tau}^{T} B_{\tau}$, that is

$$
a_{i j}^{\tau}(x)=\sum_{p=1}^{n} b_{p i}^{\tau}(x) b_{p j}^{\tau}(x)
$$

Then

$$
\sum_{i=1}^{n} \sum_{j=1}^{n}\left\|\left|a_{i j}^{\tau}(x)\right|^{1 / 80} u\right\|_{L^{2}} \leq C \sum_{p=1}^{n} \sum_{j=1}^{n}\left\|\left|b_{p j}^{\tau}(x)\right|^{1 / 40} u\right\|_{L^{2}} .
$$

Thus, combining (28), we conclude

$$
\left\|\left\langle\partial_{x} V(x)\right\rangle^{\frac{1}{20}} u\right\|_{L^{2}}+\sum_{i=1}^{n} \sum_{j=1}^{n}\left\|\left|a_{i j}^{\tau}(x)\right|^{1 / 80} u\right\|_{L^{2}} \leq C\left(\|P u\|_{L^{2}}+\|u\|_{L^{2}}\right) .
$$

The proof of Theorem 1.3 is thus complete.

\section{REFERENCES}

[1] R. Alexandre, F. Hérau and W.-X. Li, Global hypoelliptic and symbolic estimates for the linearized Boltzmann operator without angular cutoff. Preprint, arXiv:1212.4632.

[2] Y. Almog, B. Helffer, On the spectrum of non-selfadjoint Schrdinger operators with compact resolvent. Comm. Partial Differential Equations 40 (2015), no. 8, 1441-1466.

[3] P. Bolley, J. Camus and J. Nourrigat, La condition de Hörmander-Kohn pour les opérateurs pseudo-différentiels. Comm. Partial Differential Equations 7 (1982), no. 2, 197-221.

[4] F. Bouchut, Hypoelliptic regularity in kinetic equations, J. Math. Pure Appl. 81 (2002), 1135-1159.

[5] H. Chen, W.-X. Li and C.-J. Xu, Gevrey hypoellipticity for linear and non-linear Fokker-Planck equations. J. Differential Equations 246 (2009), no. 1, 320-339

[6] L. Desvillettes and C. Villani, On the trend to global equilibrium in spatially inhomogeneous entropy-dissipating systems: the linear Fokker-Planck equation, Comm. Pure Appl. Math. 54 (2001), no. 1, 1-42.

[7] B. Helffer and F. Nier, "Hypoelliptic estimates and spectral theory for Fokker-Planck operators and Witten Laplacians", Lecture Notes in Mathematics, vol. 1862, Springer-Verlag, Berlin, 2005.

[8] B. Helffer and J. Nourrigat, "Hypoellipticité maximale pour des opérateurs polynômes de champs de vecteurs", Progress in Mathematics, vol. 58, Birkhäuser Boston Inc., 1985.

[9] F. Hérau and L. Thomann, On global existence and trend to the equilibrium for the Vlasov-Poisson-Fokker-Planck system with exterior confining potential. Preprint, arXiv:1505.01698.

[10] F. Hérau, Short and long time behavior of the Fokker-Planck equation in a confining potential and applications. J. Funct. Anal. 244 (2007), no. 1, 95-118.

[11] F. Hérau, Hypocoercivity and exponential time decay for the linear inhomogeneous relaxation Boltzmann equation. Asymptot. Anal. 46 (2006), no. 3-4, 349-359.

[12] F. Hérau and W.-X. Li, Global hypoelliptic estimates for Landau-type operator with external potential. Kyoto J. Math. 53 (2013), 533-565

[13] F. Hérau and F. Nier, Isotropic hypoellipticity and trend to equilibrium for the Fokker-Planck equation with a high-degree potential, Arch. Ration. Mech. Anal. 171 (2004), no. 2, 151-218.

[14] L. Hörmander, "The analysis of linear partial differential operators", Springer-Verlag, Berlin, 1985.

[15] N. Lerner, "Metrics on the phase space and non-selfadjoint pseudo-differential operators", Birkhäuser Verlag, Basel, 2010.

[16] N. Lerner. Personal communication

[17] W.-X. Li. Global hypoellipticity and compactness of resolvent for Fokker-Planck operator, Ann. Sc. Norm. Super. Pisa Cl. Sci. (5) 11 (2012), 789-815.

[18] F. Nier, Hypoellipticity for Fokker-Planck operators and Witten Laplacians, "Lectures on the analysis of nonlinear partial differential equations", Morningside Lect. Math., 1, Int. Press, Somerville, MA, 2012, Part 1, 31-84.

[19] F. Nier, Boundary conditions and subelliptic estimates for geometric Kramers-Fokker-Planck operators on manifolds with boundaries. arXiv 1309.5070

[20] C. Villani, Hypocoercivity, Mem. Amer. Math. Soc., 202(2009),

[21] X. P. Wang, Large-time asymptotics of solutions to the Kramers-Fokker-Planck equation with a short-range potential. Comm. Math. Phys. 336 (2015), no. 3, 1435-1471. 
Wei-Xi Li, School of Mathematics and Statistics, and Computational Science Hubei Key Laboratory, Wuhan University, 430072 Wuhan, China,

AND

The Institute of Mathematical Sciences, The Chinese University of Hong Kong, Shatin, NT, Hong KONG

E-mail address: wei-xi.li@whu.edu.cn 